\title{
Stability of M13 Phage in Organic Solvents
}

\author{
Pouya Moghimian', Vesna Srot ${ }^{1}$, Benoit P. Pichon², Sandra J. Facey³, Peter A. van Aken ${ }^{1}$ \\ ${ }^{1}$ Stuttgart Center for Electron Microscopy, Max Planck Institute for Solid State Research, Stuttgart, Germany \\ ${ }^{2}$ Institut de Physique et Chimie des Matériaux de Strasbourg, UMR 7504 CNRS-Université de Strasbourg, \\ Strasbourg, France \\ ${ }^{3}$ Institute of Technical Biochemistry, University of Stuttgart, Stuttgart, Germany \\ Email: p.moghimian@fkf.mpg.de,p.vanaken@fkf.mpg.de
}

Received 25 February 2016; accepted 11 April 2016; published 14 April 2016

Copyright (C) 2016 by authors and Scientific Research Publishing Inc.

This work is licensed under the Creative Commons Attribution International License (CC BY).

http://creativecommons.org/licenses/by/4.0/

(c) (i) Open Access

\begin{abstract}
The effect of different types of organic solvents on the structural integrity of M13 phages has been directly visualized by transmission and scanning electron microscopy. The exposure of M13 phages to apolar hexane had no effect on the structure of the phages for up to $8 \mathrm{~h}$. In contrast, phages showed $\sim 10$-fold contraction into rod-like I-forms and to flattened spheroids with $\sim 12 \mathrm{~nm}$ diameter upon exposure to polar organic solvents. We show that this finding can be beneficial for the macromolecular self-assembly and in broader aspects, to enhance the spatial arrangement of desired inorganic nanoparticles in the rapidly developing field of virotronics.
\end{abstract}

\section{Keywords}

\section{M13 Phage, Organic Solvent, Structural Integrity, Self-Assembly, Electron Microscopy}

\section{Introduction}

M13 phages are being widely used in the novel virus-based technology (i.e. virotronics) and also as model systems to study liquid crystal textures due to their high aspect ratio and monodispersity [1] [2]. Viruses normally suffer from chemical aggression rather than mechanical stresses [3]. Therefore, several studies have been devoted to stress the key role of organic solvents on protein folding and inactivation of viruses [4] [5], yet it has been shown that different kinds of filamentous viruses, even from the same class, show different structural integrity in an organic solvent [6]. For instance, the filamentous phage Pf1 was inactivated in diethyl ether and acetone, whereas the filamentous phage $f d$ retained its native structure in these solvents [7]. The M13 phage is an $880 \mathrm{~nm}$ long and $6.6 \mathrm{~nm}$ wide semi-flexible virus consisting of 2700 copies of the major coat protein (pVIII) surrounding a single-stranded DNA [8]. They are among the phages being successfully used in templating inorganic nanoparticles and hence in the development of optical biosensors and battery devices [9]-[11]. The major 
coat protein is comprised of 50 amino acid residues with a highly hydrophobic core that accounts for the interlock of the coat protein with its subunits [12] [13]. It has been reported that these hydrophobic interactions have a strong role in the stabilization of virions against denaturation [7].

M13 filaments are highly sensitive to ultrasonication [13] and their worm-like shape would change to a mucus-like structure under near-infrared laser irradiations [8]. The denaturation process in solutions has been examined and observed in aqueous-organic mixtures. But viruses could experience a higher degree of denaturation in pure organic solvents. Solvent properties such as hydrophobicity and hydrogen-bonding capacity have a high impact on the structural integrity of proteins and virus particles [1] [4]. For many applications in virotronics, viruses must be stable for a certain period of time under a wide range of conditions-in particular, in different organic solvents. These solvents are often used in the production of organic electronic devices that are fabricated by solution processing (e.g. organic light-emitting diodes) [14]. While a number of studies have been devoted to the structural stability of $f d$ [15], f1 [7] and T7 [3] phages in organic solvents, no work has been performed yet to systematically study the structural integrity of M13 phages in different organic solvents. Establishing conditions under which M13 phages are stable is an important determinant in the practical utility of the virus technology [1].

Here, we expose M13 filamentous phages to organic solvents and report their dissolution behavior and conformational changes using transmission electron microscopy (TEM) and scanning electron microscopy (SEM). The aim is to find an appropriate organic solvent, in which the virions can retain their original structure and can maintain their directional assembly for longer periods of time.

\section{Materials and Methods}

Wild-type (WT) M13 phage (M13KE) was purchased from New England Biolabs, Inc. Phage propagation and purification have been performed according to our previously reported method [16]. The WT M13 phages were suspended in Tris (hydroxymethyl) aminomethane (TBS) at $\mathrm{pH}$ 7.4. An aliquot of phage solution (5 $\mu \mathrm{l}$ ) with a concentration of $1.4 \times 10^{12} \mathrm{pfu} / \mathrm{ml}$ was added to an equal volume of an organic solvent (hexane (Sigma-Aldrich), chloroform (Merck), tetrahydrofuran (THF, Merck) and toluene (Sigma-Aldrich)) at room temperature. The mixture was left unstirred for $60 \mathrm{~min}$. Thereafter, a $5 \mu \mathrm{l}$ droplet of the mixture was loaded for 5 min onto Quantifoil $^{\circledR}$ (Quantifoil Micro Tools GmbH) TEM grids covered by a carbon support film. The samples were gently washed with Milli-Q water, negatively stained with $1 \%$ uranyl acetate (UA) and blotted dry. The Zeiss SESAM microscope, a $200 \mathrm{kV}$ FEG microscope was used to carry out TEM investigations. The average viral particle sizes were calculated by averaging sizes over all the particles of the same type on the acquired bright-field (BF)-TEM images. The SEM images were acquired by a Zeiss DSM 982 Gemini operating at $3 \mathrm{kV}$. To assemble a homogenous and closely-packed viral film on silicon wafers, the viral particles were directionally deposited using the convective assembly technique by pulling a meniscus containing $5.5 \mu \mathrm{l}$ of the virus solution across the substrate at a constant rate $(1.2 \mathrm{~mm} / \mathrm{min})$.

\section{Results and Discussion}

Untreated phages were included in this study as a control sample (Figure 1(a), Figure 1(b)). BF-TEM observations of hexane-treated M13 phages revealed that the virions retained their structure, flexibility and original dimensions (see Figure 1(c), Figure 1(d)). Phages showed no decomposition or fragmentation after $1 \mathrm{~h}$ contact with hexane. This could be associated with the fact that hexane is a nonpolar hydrophobic solvent that cannot compete for hydrogen bonds between proteins and thus was not able to denature the native structure of the phages. However, when exposed to chloroform, phages were destabilized from their native structure and they were $\sim 5$-fold contracted into shortened I-form rods having an average length of $\sim 0.18 \mu \mathrm{m}$ (blue arrows in Figure 1(e)). A rather low portion of spherical filaments (spheroids or S-forms) having an average diameter of $\sim 17$ $\mathrm{nm}$ were also present (red arrows in Figure 1(f)). Both morphological changes have been reported to result in the inactivation (i.e. loss of infectivity) of phages [7] [15] [17] [18]. The reason for these observations relies on the fact that chloroform is a polar solvent and is therefore able to break the hydrogen bonding and the hydrophobic interactions between the capsid proteins [17].

Similarly, BF-TEM images of THF-treated phages showed apparent fragmentation of the phages but in a greater extend (red and blue arrows in Figure 1(g), Figure 1(h)). The original filaments were extremely decomposed mostly into S-forms and in a less amount in I-forms. This could be a result of peptide bonds breaking 

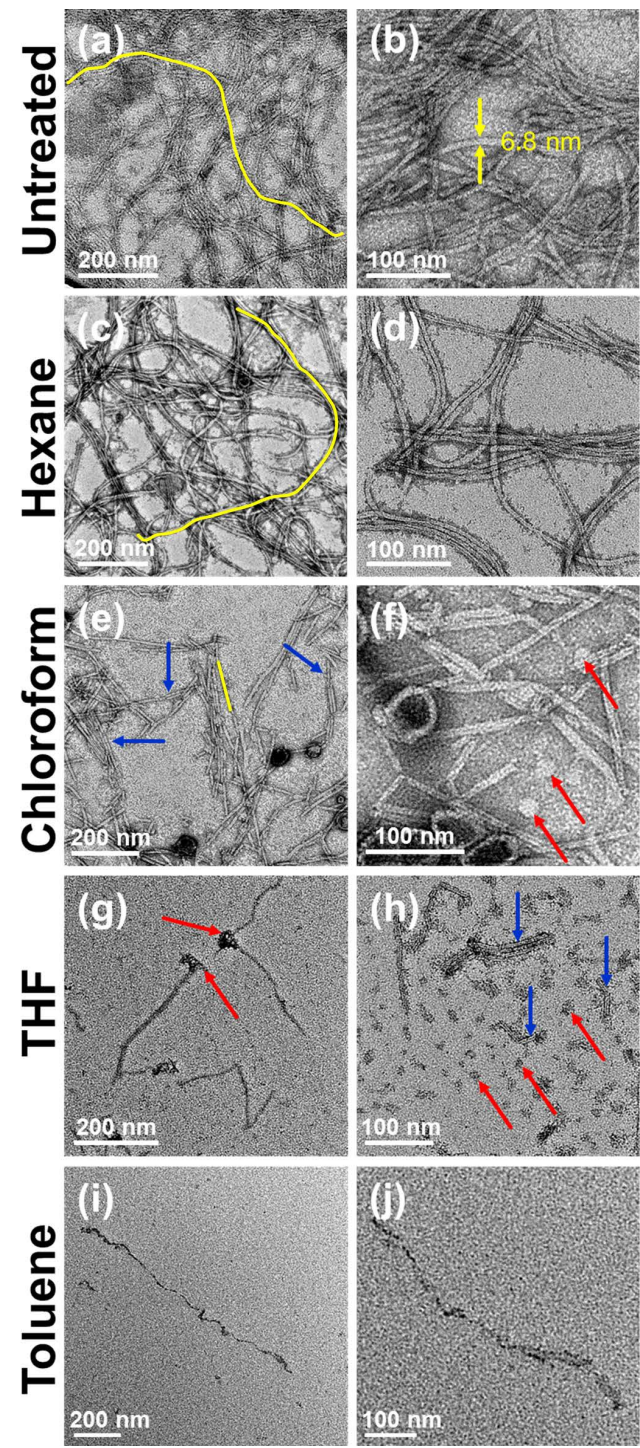

Figure 1. BF-TEM images of untreated M13 phages (a) (b) and the conformational and structural changes of phages after $1 \mathrm{~h}$ exposure to (c) (d) hexane, (e) (f) chloro-form, (g) (h) THF and (i) (j) toluene. The right column shows high-magnification BF-TEM images. Yellow lines mark an intact phage in (a) (b) and a single shortened I-form rod in (e) as a means of identification. Blue and red arrows show the contraction of M13 intact filaments to I-forms (shortened rods) and spheroids, respectively.

in capsid proteins and by having the coat proteins detached from the core DNA which had been held together by weak electrostatic interactions [17]. The diameter of rods and spheroids were in average $\sim 0.12 \mu \mathrm{m}$ and $\sim 13 \mathrm{~nm}$, respectively, which shows more filament contraction ( 10-fold contraction) and decomposition rate compared to the chloroform-treated filaments. THF is one of the most polar ethers and has a similar polarity as chloroform. However, THF is hydrophilic and water-miscible and therefore it is able to dissolve in the phage solution and rapidly break the hydrogen bonds in proteins and therefore changes the structure. In contrast, chloroform is hydrophobic and does not have as much interaction with the phages as THF. This could be the reason that in our specific experimental time period, phages showed a higher degree of decomposition in THF compared with that in chloroform. It should be noted that this phenomenon is a function of exposure time, indicating that phages would suffer the same level of decomposition in THF after a certain amount of time as they did in chloroform. In contrast to chloroform and THF, toluene has a relatively low polarity and therefore did not show high fragmentation of M13 filaments, yet was able to deform the phages to a certain level. As shown in Figure 1(i), Fig- 
ure $1(\mathrm{j})$, no I-form or S-form particles could be observed, however virions were kinked which could be attributed to the disruption of the molecular tertiary structure interactions and deformation of the capsid arrangement [4]. Hence, the solvent polarity has a significant contribution to the interactions that lead to the structural changes of M13 phages. Higher polarities of the solvents employed in this study have a higher influence on breaking inter-coat protein forces, and weakening the van der Waals interactions between the DNA and the coat proteins.

Next, we assembled a well-aligned film of phages on a silicon wafer (Figure 2(a), Figure 2(c)) and immersed it for $8 \mathrm{~h}$ in $3 \mathrm{ml}$ of hexane. As shown in Figure 2(b), Figure 2(d), the hexane immersion did not disrupt the phage assembly even up to $8 \mathrm{~h}$, showing that extended exposures to hexane has no influence not only on the structural integrity of virions, but also on the their directional assembly. The alignment of the phage assemblies can be identified by 2D Fourier transform (FT) images. The insets in Figure 2(a), Figure 2(b) show the FT images with an intensity pattern in a narrow and elongated ellipse perpendicular to the direction of alignment, that confirms the highly-aligned structure of the phage assemblies.
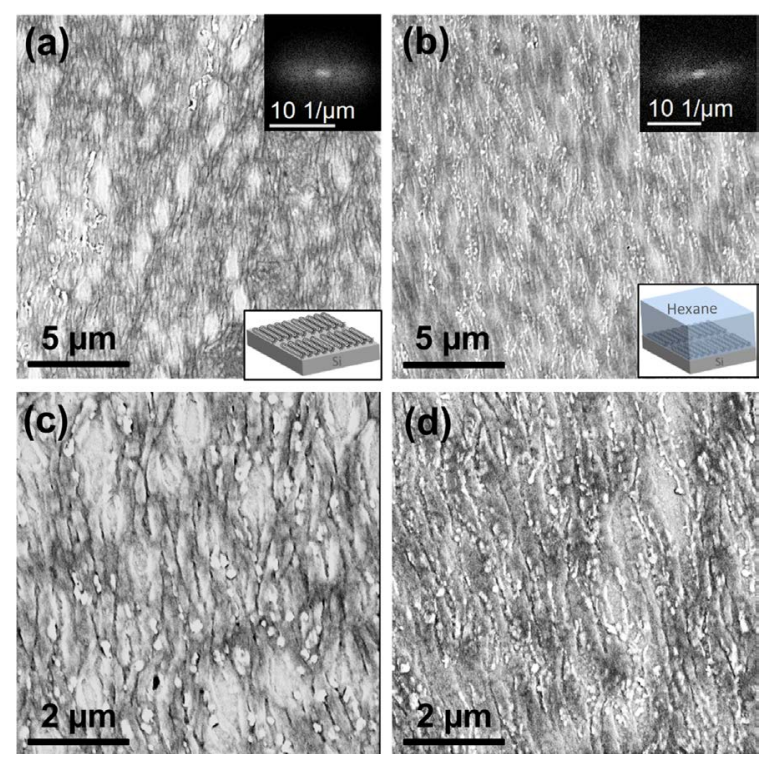

\begin{abstract}
Figure 2. SEM image of a densely-packed and well-aligned assembly of phages on (a) silicon substrate and (b) after exposure of the assembly to hexane for $8 \mathrm{~h}$. The small white round particles on the top of the phage layers are composed of sodium $(\mathrm{Na})$ and chlorine $(\mathrm{Cl})$ (data not shown here) which arise from the salts present in the primary phage solution. The insets show the 2D FT images of the corresponding SEM images. (c) and (d) High-magnification images of (a) and (b), respectively.
\end{abstract}

This key finding is of high importance in virotronics, in which viruses are used as unique biological entities to create advanced multi-component materials with a high molecular-level control [19] [20]. The functional properties of such devices, e.g. electrical [21] [22] and magnetic collective properties [23] can be maximized by using a self-assembled layer of organic molecules to spatially arrange inorganic nanostructures [24]. However, inorganic nanoparticles usually come in contact with organic templates in the presence of an organic solvent. Therefore, hexane can be a great candidate for a dispersion medium for the desired inorganic particles prior to them assembling into functional 2D films.

\title{
4. Conclusion
}

In closing, this study provides direct assessment of the stability of the filamentous M13 structure when exposed to different organic solvents. Efforts to maintain the structural integrity of viruses lead to an increased understanding of the factors that disrupt their structure. Phages showed to be denatured in polar organic solvents as compared to nonpolar solvents. This phenomenon could be understood by considering three factors: solvent hydrogen-binding capacity, hydrophobicity and water miscibility. This work also gives information on the handling of supramolecular assemblies in organic solvents for virus-based materials. This can be used in the devel- 
oping topic of research that is the control of macromolecular assemblies with hierarchical frameworks.

\section{Acknowledgements}

The financial support by the DFG is gratefully acknowledged. The research leading to these results has received funding from the European Union Seventh Framework Programme [FP7/2007-2013] under grant agreement no. 312483 (ESTEEM2). P. M. thanks Dr. V. Vyas (MPI-FKF, Stuttgart, Germany) for discussions and critical reading of the manuscript and C. Mollenhauer (MPI-IS, Stuttgart, Germany) for providing organic solvents.

\section{References}

[1] Nasir, S.F. and Jaworski, J. (2014) Assessing the Stability of Assembled Filamentous Phage Coat Protein P8. Supramolecular Chemistry, 26, 329-337. http://dx.doi.org/10.1080/10610278.2013.835813

[2] Lee, S.W., Mao, C.B., Flynn, C.E. and Belcher, A.M. (2002) Ordering of Quantum Dots Using Genetically Engineered Viruses. Science, 296, 892-895. http://dx.doi.org/10.1126/science.1068054

[3] Hernando-Perez, M., Pascual, E., Aznar, M., Ionel, A., Caston, J.R., Luque, A., et al. (2014) The Interplay between Mechanics and Stability of Viral Cages. Nanoscale, 6, 2702-2709. http://dx.doi.org/10.1039/c3nr05763a

[4] Mattos, C. and Ringe, D. (2001) Proteins in Organic Solvents. Current Opinion in Structural Biology, 11, $761-764$. http://dx.doi.org/10.1016/S0959-440X(01)00278-0

[5] Lau, F.W. and Bowie, J.U. (1997) A Method for Assessing the Stability of a Membrane Protein. Biochemistry, 36, 5884-5892. http://dx.doi.org/10.1021/bi963095j

[6] Amako, K. and Yasunaka, K. (1977) Ether Induced Morphological Alteration of Pf-1 Filamentous Phage. Nature, 267, 862-863. http://dx.doi.org/10.1038/267862a0

[7] Olofsson, L., Ankarloo, J., Andersson, P.O. and Nicholls, I.A. (2001) Filamentous Bacteriophage Stability in NonAqueous Media. Chemistry \& Biology, 8, 661-671. http://dx.doi.org/10.1016/S1074-5521(01)00041-2

[8] Tsen, K.T., Tsen, S.-W.D., Fu, Q., Lindsay, S.M., Kibler, K., Jacobs, B., et al. (2010) Photonic Approach to the Selective Inactivation of Viruses with a Near-Infrared Ultrashort Pulsed Laser. SPIE Proceedings, 7561, 75610W-75610. http://dx.doi.org/10.1117/12.841316

[9] Mao, C., Liu, A. and Cao, B. (2009) Virus-Based Chemical and Biological Sensing. Angewandte Chemie International Edition, 48, 6790-6810. http://dx.doi.org/10.1002/anie.200900231

[10] Lee, Y.J., Yi, H., Kim, W.-J., Kang, K., Yun, D.S., Strano, M.S., et al. (2009) Fabricating Genetically Engineered HighPower Lithium-Ion Batteries Using Multiple Virus Genes. Science, 324, 1051-1055. http://dx.doi.org/10.1126/science.1171541

[11] Lee, Y.M., Kim, Y.H., Lee, J.H., Park, J.H., Park, N.-G., Choe, W.-S., et al. (2011) Highly Interconnected Porous Electrodes for Dye-Sensitized Solar Cells Using Viruses as a Sacrificial Template. Advanced Functional Materials, 21, 1160-1167. http://dx.doi.org/10.1002/adfm.201001774

[12] Johnson, I.D. and Hudson, B.S. (1989) Environmental Modulation of M13 Coat Protein Tryptophan Fluorescence Dynamics. Biochemistry, 28, 6392-6400. http://dx.doi.org/10.1021/bi00441a035

[13] Stopar, D., Spruijt, R.B., Wolfs, C. and Hemminga, M.A. (1998) Mimicking Initial Interactions of Bacteriophage M13 Coat Protein Disassembly in Model Membrane Systems. Biochemistry, 37, 10181-10187. http://dx.doi.org/10.1021/bi9718144

[14] Zhong, C., Duan, C., Huang, F., Wu, H. and Cao, Y. (2011) Materials and Devices toward Fully Solution Processable Organic Light-Emitting Diodes. Chemistry of Materials, 23, 326-340. http://dx.doi.org/10.1021/cm101937p

[15] Roberts, L.M. and Dunker, A.K. (1993) Structural Changes Accompanying Chloroform-Induced Contraction of the Filamentous Phage fd. Biochemistry, 32, 10479-10488. http://dx.doi.org/10.1021/bi00090a026

[16] Moghimian, P., Srot, V., Rothenstein, D., Facey, S.J., Harnau, L., Hauer, B., et al. (2014) Adsorption and Self-Assembly of M13 Phage into Directionally Organized Structures on C and $\mathrm{SiO}_{2}$ Films. Langmuir, 30, 11428-11432. http://dx.doi.org/10.1021/la502534t

[17] Wigle, J.C., Holwitt, E.A., Noojin, G.D., Estlack, L.E., Sheldon, K.E. and Rockwell, B.A. (2011) No Effect of Femtosecond Laser Pulses on DNA, Protein, M13, or E. coli. SPIE Proceedings, 7897, 789716-789719. http://dx.doi.org/10.1117/12.874754

[18] Martinek, K., Mozhaev, V.V., Smirnov, M.D. and Berezin, L.V. (1980) Reactivation of "Irreversibly” Denaturated Enzymes. Biotechnology and Bioengineering, 22, 247-251. http://dx.doi.org/10.1002/bit.260220121

[19] Chung, W.J., Sena, M., Merzlyak, A. and Lee, S.W. (2011) Phages as Tools for Functional Nanomaterials Develop- 
ment. In: Paul, D., Ed., Comprehensive Biomaterials, Elsevier, Oxford, 95-111. http://dx.doi.org/10.1016/B978-0-08-055294-1.00064-7

[20] Moghimian, P., Kilper, S., Srot, V., Rothenstein, D., Facey, S.J., Hauer, B., et al. (2016) Phage-Assisted Assembly of Organic-Inorganic Hybrid Bilayers. International Journal of Materials Research, 107, 295-299. http://dx.doi.org/10.3139/146.111351

[21] Yang, S.H., Chung, W.J., McFarland, S. and Lee, S.W. (2013) Assembly of Bacteriophage into Functional Materials. Chemical Record, 13, 43-59. http://dx.doi.org/10.1002/tcr.201200012

[22] Lee, B.Y., Zhang, J., Zueger, C., Chung, W.-J., Yoo, S.Y., Wang, E., et al. (2012) Virus-Based Piezoelectric Energy Generation. Nature Nanotechnology, 7, 351-356. http://dx.doi.org/10.1038/nnano.2012.69

[23] Toulemon, D., Pichon, B.P., Cattoen, X., Man, M.W.C. and Begin-Colin, S. (2011) 2D Assembly of Non-Interacting Magnetic Iron Oxide Nanoparticles via “Click” Chemistry. Chemical Communications, 47, 11954-11956. http://dx.doi.org/10.1039/c1cc14661k

[24] Pichon, B.P., Demortière, A., Pauly, M., Mougin, K., Derory, A. and Bégin-Colin, S. (2010) 2D Assembling of Magnetic Iron Oxide Nanoparticles Promoted by SAMs Used as Well-Addressed Surfaces. The Journal of Physical Chemistry C, 114, 9041-9048. http://dx.doi.org/10.1021/jp101872u 